\title{
Relationship among Satisfaction with Life, Family Care Degree, Psychological Dependency and Subjective Bias of Senior High School Student
}

\author{
Ying Guo, Xia Chen \\ College of Teacher Education and Psychology, Sichuan Normal University, Chengdu, China \\ Email: guoying517@163.com
}

Received 22 February 2016; accepted 27 March 2016; published 30 March 2016

Copyright (C) 2016 by authors and Scientific Research Publishing Inc.

This work is licensed under the Creative Commons Attribution International License (CC BY). http://creativecommons.org/licenses/by/4.0/

\section{Abstract}

The purpose of the present study was to learn the relationship among satisfaction with life, family care degree, psychological dependency and subjective bias of senior high school student. We are using the Life Satisfaction Scale (LSIB), Family Care Scale (APGAR), Dependent Scale (Dy), Prejudice Scale (Pr), for 300 high school students in the school randomly sampling survey. Life satisfaction was investigated with the APGAR test, psychological dependence, prejudice relationship personality. Through this study, we have drawn the following results: 1) Life satisfaction was significantly positively correlated with the degree of care to the family $(r=0.311, p<0.01)$, and psychological dependence $(r=-0.399, p<0.01)$, subjective bias negative correlation $(r=-0.328, p<$ 0.01). APGAR and dependence $(r=-0.147, p<0.05)$, biased were negatively correlated $(r=-0.134$, $p<0.05)$. Dependence and prejudice were positive correlation $(r=0.661, p<0.01) ; 2)$ as for high school students of different gender within life satisfaction, family care degree, dependency and prejudice score comparison, APGAR $t$ test was significantly $(t=-2.38, p<0.05)$; 3 ) by comparison of the scores of different professions, science students life satisfaction was higher than liberal arts students $(t=2.12, p<0.05)$; 4) family APGAR and dependence predict life satisfaction $(p<0.01)$ were greater than the subjective bias $(p=0.177)$ of forecasts. The role of the family APGAR return was greater than the return action dependent. Based on these results, we have come to the conclusion that the degree of family care and psychological dependence performance effectively predicts life satisfaction. APGAR on life satisfaction was positively predicted. Psychological dependency on life satisfaction was negative prediction.

\section{Keywords}

Senior High School Student, Life Satisfaction, APGAR, Psychological Dependence, 


\section{Subjective Bias}

\section{Introduction}

Life satisfaction are individuals with their lives overall judgment (Wang, 2006). As a cognitive factor, it affects the individual emotional experience, thus affects the individual pursuit of life goal orientation and behavior orientation, and plays an important role for the individual and social. Michael Argyle defined the life satisfaction as the overall evaluation of the quality of life. Life satisfaction is individual based on their own set of standards for the quality of life by the subjective evaluation (Yao et al., 2011). The individual is in accordance with their own choice of standard to own most of the time or the totality of the cognitive evaluation for a period of time living conditions.

At the present, domestic has begun research on adolescent life satisfaction. Researchers from many studies found that family environment, personality characteristics, self-esteem and many other factors will affect the individual life satisfaction (Ma, 2009). For example Jinxia Wang, Jichun Wang and others in a 2005 study found that the parent-child relationship satisfaction and parents marriage happiness degree evaluation are very significant effecting on senior high school students of general life satisfaction; the way of parents' emotional warmth type with senior high school students' general life satisfaction has significant positive correlation, which refused to deny and punishing upbringing; senior high school students of general life satisfaction has significant negative correlation (Wang \& Wang, 2006). These results have proved that the parent-child relationship satisfaction, parenting style of refused to deny and punishing parenting is predicting the strong factors of senior high school students' general life satisfaction. Again as in 2007, Lili Tian, Xue Zheng and others whose were on five factors of personality and life satisfaction relationship found that students of personal privacy are closely related to life satisfaction of senior high school students (Tian \& Zheng, 2008). Time to push forward, as in 2004 Lina Chen, Jianxin Zhang and others, on college students' general life satisfaction and its relationship with self-esteem, proved that most of the students to study in school are moderately satisfied with their life and their life satisfaction in terms of gender and area are difference obvious. High level of self-esteem as a part of the student's life satisfaction is also higher (Chen \& Zhang, 2005). But previous research on life satisfaction is inclined to the elderly residents. Student's life satisfaction is lack of empirical research, so we can only get a few theoretical data from previous studies and a lot of problems to be further empirical research.

Positive psychology is an important trend of contemporary psychology, and life satisfaction as a part of the composition of positive psychology, inevitably becomes a hot research topic of contemporary psychology. This has implementation both in the field of psychology and the sociology with the common direction of development and changes of requirements, and psychology achieve to lead people to the happiness of study and life which is the inevitable way to this historical mission.

Family as an important part of the process of personal growth environment, for the formation and development of individual self system and individual socialization, provides the necessary physical and emotions environment. As parents have only one children, the negative impact of excessive care children should cause parents to recognize and vigilant (Hai, 2007). In terms of the caregivers inner, the excessive concern is actually a strong need to others, that is psychological dependence. It belongs to a kind of unhealthy psychology. Dependency and prejudices are a part of the personality. Through the care of family and life satisfaction, dependency and prejudice can help us to explore the interaction relationship between them. And it helps us to take certain measures to correct the bad personality for high school students, and parents give us a right way of education.

\section{Methods}

\subsection{Participants}

Using simple random sampling method. All objects derived from a senior high school of Sichuan province. A total of 300 people involved in the investigation object. We have hand out 300 questionnaires, eliminating invalid questionnaire 37 , the effective recovery of 263 , the recovery rate was $86.67 \%$. Including 205 senior one student, senior two 58 people, 129 boys, 134 girls. Age range between 15 to 18, average (16.20 - 0.811). All 
subjects in line with the principle of voluntary and they attended this tests with in clear of the nature of the test paper and purpose.

\subsection{Research Tools}

\subsubsection{Life Satisfaction Rating Scale (LSIB)}

Life Satisfaction Scale (Life Satisfaction Rating Scale, LSR) was formulated by American Neugarten and others which is all of Life Satisfaction Scale for one of them (Miao, 2007). This is his rating scale. This study is life satisfaction degree B from the rating scale (life satisfaction degree B, LSIB). A total of 12 questions of the scale, are from the lowest satisfaction score 0 to the highest degree of satisfaction of 22. The performance of life satisfaction was in turn from the lowest to the highest. LSIB and LSR consistency are 0.58 and 0.55 ; It is consistent with the evaluation of a clinical psychologist at 0.47 and 0.39 respectively. The consistency of LSIA and LSIB is 0.73 (Zhao \& Xi, 2011).

\subsubsection{Dependence Scale (Dy)}

Navran according to related research finally picked from the MMPI 57 topics, including 49 a "is" score, score 8 answer "no" and Birtchnell on 6 groups of different individual study found that, from the gender, women easy entry of the scale and dependent scale (Dy) score is not affected by age (Zhang, 2005). ShuMao Jiand others through the study also found that age didn't seem to affect the change of Dy scores, but women usually have higher scores than men do. Adult men and women in our country Dy questionnaire correlation coefficient between 4 - 6 weeks heavy test are: 0.67 and 0.81 . Low marks have good psychological adaptability, autonomy, which feeling can be understood, happy and the body without discomfort. High marks suggest that the mental is maladjustment and dependence is strong and often feel misunderstood, unpleasant, body uncomfortable (Zhang, 2005).

\subsubsection{Prejudice Scale (Pr)}

Gough according to the research of the high school students develop the bias of Scale. The scale are total of 32 subjects, including a "is" scoring 29 item, three entries to answer "no" scoring (Zhang, 2005). Scores were rarely influenced by age and gender. The scale of the retest reliability is high, which reflects the characteristics of the fairly stable personality. The people whose self assessment and problem solving low efficiency have higher score. The people whose efficiently and agile thinking have lower score. Pr scores elevated prompt subjects usually disposition introversion, pedantic, concept of rigid, lack of flexibility. It is always emphasized their own values and reluctant to reflect on their own beliefs.

\subsubsection{Family Care Degree Scale (APGAR)}

It is total of five topics, which are by fitness, cooperation, growth degree, emotional degrees, closeness of five projects (Zhang, 2005). Grading methods for three points method, total score is 7 to 10 minutes that said family function good and 4 to 6 points said the family function moderate disabilities and family functioning serious obstacle be 0 to 3 points.

\subsection{Statistical Analyses}

We are using the SPSS (17.0) statistical software to analyze data processing. Statistical methods are including description and inference statistics. Description statistics are including the mean, standard deviation, standard error of the mean. Inference statistics are including multivariate linear correlation, t-test, independent sample t-test) and multiple linear regression analysis.

\section{Results}

There are description of the life satisfaction and family care, psychological dependence, bias statistics in the Table 1. From this we can see some preliminary analysis of the data in the table.

From Table 2 on the life satisfaction and family care, psychological dependence, the bias of the analysis of the correlation between the data we can see high school students life satisfaction and family care at 0.01 level (double side) which were significantly positive correlation. Psychological dependence and bias on the 0.01 level 
Table 1. The description of the life satisfaction and family care, psychological dependence, bias statistics.

\begin{tabular}{cccc}
\hline & \multicolumn{2}{c}{ A single sample statistics } & Mean standard error \\
\hline & Mean & Standard deviation & 0.206 \\
\hline Life satisfaction & 10.45 & 3.344 & 0.170 \\
Familycare degree & 5.10 & 2.759 & 0.284 \\
Subjective bias & 14.99 & 4.603 & 0.526 \\
Dependency & 31.06 & 8.527 & \\
\hline
\end{tabular}

Table 2. Life satisfaction and family care, psychological dependence, the bias of the analysis of the correlation.

\begin{tabular}{|c|c|c|c|c|c|}
\hline & & Life satisfaction & Family care degree & Prejudice & Dependency \\
\hline \multirow{3}{*}{ Life satisfaction } & Pearson correlation & 1 & $0.311^{* *}$ & $-0.328^{* *}$ & $-0.399^{* *}$ \\
\hline & Significant (both sides) & & 0.000 & 0.000 & 0.000 \\
\hline & $\mathrm{N}$ & 263 & 263 & 263 & 263 \\
\hline \multirow{3}{*}{ Family care degree } & Pearson correlation & $0.311^{* *}$ & 1 & $-0.134^{*}$ & $-0.147^{*}$ \\
\hline & Significant (both sides) & 0.000 & & 0.030 & 0.017 \\
\hline & $\mathrm{N}$ & 263 & 263 & 263 & 263 \\
\hline \multirow{3}{*}{ Subjective bias } & Pearson correlation & $-0.328^{* *}$ & $-0.134^{*}$ & 1 & $0.661^{* *}$ \\
\hline & Significant (both sides) & 0.000 & 0.030 & & 0.000 \\
\hline & $\mathrm{N}$ & 263 & 263 & 263 & 263 \\
\hline \multirow{3}{*}{ Dependency } & Pearson correlation & $-0.399^{* *}$ & $-0.147^{*}$ & $0.661^{* *}$ & 1 \\
\hline & Significant (both sides) & 0.000 & 0.017 & 0.000 & \\
\hline & $\mathrm{N}$ & 263 & 263 & 263 & 263 \\
\hline
\end{tabular}

Note: ${ }^{* *}$ at 0.01 level (double side); ${ }^{*}$ at 0.05 level (double side).

(both sides) were significantly negative correlation relationship. Family care and life satisfaction is also on the 0.01 level (double side) which was positively related to relationship, family care and psychological dependence. Subjective bias on the 0.05 level (double side) was significantly negative correlation relationship. Subjective bias and dependence on the 0.01 level (both sides) were positively correlated. Gender as independent variables, respectively by high school students life satisfaction, family care, psychological dependence, bias as a dependent variable for independent samples $t$ test, the results are shown in Table 3 . The subjects of family care scoring are significant gender differences, $\mathrm{t}(261)=2.38, p<0.05)$. Boys' family care degree are higher than the girls' in the scale score. Life satisfaction and dependence and prejudice are no gender difference on gender scores, $\mathrm{t}(261)$ $=0.23,1.08,0.97, p>0.05$ ). In professional quality for grouping variable, respectively by high school students life satisfaction, family care, dependency, subjective bias to examine variables for independent samples t test, the results are shown in Table 4. We are analysis the subjects life satisfaction significant differences in different professional score, $\mathrm{t}(261)=2.12, p<0.05$. The school of science living life satisfaction was significantly higher than that of the school of arts living life satisfaction. Test results also shows that the different professional high school students in the family care, dependence and subjective bias have no significant difference, $\mathrm{t}(261)=0.56$, $0.80,0.42, p>0.05$ ). Specific results are shown in Table 4. In order to further investigate which factors significantly affect high school students life satisfaction, we put the life satisfaction as dependent variable, the degree of family care and dependence, and bias as independent variables multiple linear regression analysis. According to the results we can draw the family care degree $(p<0.01)$ and dependence $(p<0.01)$ influence on life satisfaction significantly greater bias $(p>0.05)$. The results also illustrates that the degree of family care and prejudice on life satisfaction have significant prediction effect. Finally use the stepwise regression analysis degree of family care and dependent contribution to life satisfaction, respectively, and the results are as shown in Table 5. The 
Table 3. Comparison of different gender students life satisfaction and family care, dependency, bias score.

\begin{tabular}{cccc}
\hline & Girl & Boy & $t(261)$ \\
\hline Life satisfaction & $10.46 \pm 3.19$ & $10.45 \pm 3.49$ & 0.23 \\
Family care degree & $5.42 \pm 2.69$ & $4.78 \pm 2.79$ & $-2.38^{*}$ \\
Dependency & $14.96 \pm 4.57$ & $15.02 \pm 4.65$ & -1.08 \\
Subjective bias & $30.54 \pm 8.71$ & $31.57 \pm 8.34$ & -0.97 \\
\hline
\end{tabular}

Note: ${ }^{*} p<0.05$.

Table 4. Comparison of different professional high school life satisfaction and family care, dependency, bias score.

\begin{tabular}{cccc}
\hline & Science & Arts & $t(261)$ \\
\hline Life satisfaction & $10.63 \pm 3.36$ & $9.94 \pm 2.28$ & $2.12^{*}$ \\
Family care degree & $5.16 \pm 2.70$ & $4.94 \pm 2.91$ & 0.56 \\
Dependency & $14.86 \pm 4.37$ & $15.38 \pm 5.19$ & -0.80 \\
Subjective bias & $31.05 \pm 8.30$ & $31.10 \pm 9.18$ & -0.42 \\
\hline
\end{tabular}

Note: ${ }^{*} p<0.05$.

Table 5. Degree of family care and dependence on life satisfaction of stepwise regression analysis.

\begin{tabular}{|c|c|c|c|c|c|c|c|c|}
\hline \multicolumn{9}{|c|}{ Coefficient $^{\mathrm{a}}$} \\
\hline & \multirow{2}{*}{ Model } & \multicolumn{2}{|c|}{$\begin{array}{l}\text { Unstandardized } \\
\text { coefficients }\end{array}$} & \multirow{2}{*}{$\begin{array}{c}\text { Standardized } \\
\text { coefficients }\end{array}$} & \multirow[t]{2}{*}{$\mathrm{t}$} & \multirow{2}{*}{ Sig. } & \multicolumn{2}{|c|}{$\begin{array}{c}\text { Confidence interval } \\
\text { with B }(95.0 \%)\end{array}$} \\
\hline & & B & SE & & & & Down & Up \\
\hline \multirow{3}{*}{1} & (Constant) & 13.252 & 0.818 & & 16.194 & 0.000 & 11.640 & 14.863 \\
\hline & Family care degree & 0.313 & 0.067 & 0.258 & 4.679 & 0.000 & 0.181 & 0.445 \\
\hline & Dependency & -0.142 & 0.022 & -0.361 & -6.538 & 0.000 & -0.184 & -0.099 \\
\hline
\end{tabular}

Note: ${ }^{a}$ Dependent variable: life satisfaction.

regression equation is $y=13.252+0.313 x_{1}-0.142 x_{2}$. The interpretation of the equations is 13.252 according to the equation of the straight line in the y intercept is 13.252. Family care is unchanged, the prejudice change a unit, the life satisfaction will change 0.142 units; When the bias is constant, the degree of family care change a unit, the life satisfaction will change 0.313 units.

\section{Discussion}

\subsection{Life Satisfaction and Family Care}

Previous studies have indicated that the college students' life satisfaction and parents upbringing have obvious correlation (Li, 2013). Parents give their children more affection and care; giving more protection and care will increase children's life satisfaction; parents too excessive interference or protection or punish severely will reduce child's life satisfaction. This study found that the average high school life satisfaction scale score of 10.45. According to the dimension of life satisfaction score, the average score of this study are somewhere in the middle. The score suggests that high school students' life satisfaction are at the average level and most of the students are not very satisfied with life now. This is a notable signal for us. In this study, the high life satisfaction score lower than the high life satisfaction evaluation, this may be because senior students just entering a new environment and also is not fully integrated into the collective, not adapt to the new life now. 
In family care questionnaire the average scored is 5.1. It shows that the population in a moderate family obstacles. Which girl's average of 5.42 is higher than boy's average of 4.78, it is illustrates that the boys on the score of evaluation of family lower than girls. In real life, parents are more concerned about education efforts of fine girl, but for boys is more with a loose rein. When girls are get in trouble they are always seek the opinions of their parents. But the boy is not do that, they solve problems independently. This creates that not only the girl is more emotional communication with their parents than boys but also on the degree of recognition and understanding of family is much higher than the boy (Peng, 2012). So the girls than boys are more satisfied the way to get along with their family. And on family care degree of each factor, the cooperation degree score (0.80) was significantly lower than fitness (0.88), growth degrees (1.09), emotional degree (1.08), intimacy (1.29). It shows that the family in the treatment of the problem and make a decision to resolve the crisis and the lack of effective experience and the way parents to deal with the problem is not flexible.

The questionnaire results suggest us that high school students have the problems of family function. Many parents migrant workers, seldom communicate with their child's learning life and they don't interfere with the studying life of children. That is to say, when children encounter problems with parents, the parents have no response or the exchange and it is also caused the children dissatisfied with the family. Another kind of circumstance is that parents just meet the needs of the children on the material. They have neglected children in spiritual need and do not know how to get along with children. This also causes children are not happy with family. In family care degree and life satisfaction of correlation analysis showed highly relevant trends. It tips us that family care degree can also affect high school students on life satisfaction evaluation. Research shows that if the parents in finance, responsibility, raising children, individual character and family activities don't reach the consensus of opinion or don't care about the child's inner thoughts, it is easy to cause the child are not satisfied with life (He \& Huang, 2009). Family and life are inseparable.

\subsection{The Interpretation of the Life Satisfaction and Prejudices and Dependency}

In prejudice and dependence scale, the results show that the bias score is not affected by age and gender and dependency scale is not affected by age. But dependent on the score of girls (31.57) is higher than that of boys (30.54), it shows that girls than boys more agree with this scale, which is consistent with the predecessors' research results. Dependence of scores of girls higher than boys can be interpreted as girls of independence were worse than boys, which resulted in the girl in many ways. For example girls can not solve problems independently and need to rely on the help of others

\subsection{Related to Life Satisfaction}

In the related research, life satisfaction and the degree of family care, psychological dependence and subjective bias are highly related. This is suggests that our family care and psychological dependence, as well as the subjective bias, is one of the factors that affect life satisfaction. Life satisfaction and family care degree show positive correlation trend, it shows that the higher the evaluation of the individual to the family, the higher the evaluation of life satisfaction. And dependency and prejudice all showed a trend of obvious negative correlation, which shows that the stronger the individual is, the higher the score of the individual is not satisfied with the life. Because of the life satisfaction and the family care degree of the influence factors deriving from life, the family factor is an important factor affecting life satisfaction and the individual life satisfaction evaluation also indirectly reflects part of the care of the family. This is consistent with the study of theoretical assumptions. In the study of life satisfaction in different professional high school students found that high school of science living score (10.63) is higher than that of the school of arts living score (9.94). Generally speaking science students are thinking more straightforward, regardless of the gain and loss. The school of arts living idea is exquisite, which is care about also than science more. So in life, the student of science can meet in life easily more than arts and they can accept more difficulty and the test of life.

\subsection{Prediction of Life Satisfaction}

In family care and dependency and prejudice on the life satisfaction of regression analysis, the research results show that degree of family care and dependence have linear regression significant on life satisfaction, this show that the degree of family care and dependency can predict life satisfaction to a certain extent. Existing studies 
have shown that the influencing factors of life satisfaction included family factors and the individual in their life satisfaction evaluation was from the environment. Family also is the environment that we will experience in any period of our life, so the family of life satisfaction of individual environmental impact evaluation is accord with actual conditions. In this study, the higher the score of the individual family care degree of the higher life satisfaction of evaluation, this means that individual's family environment better, the individual to the family as it certainly sure or family to individual, then life satisfaction evaluation will be more and more certain. In addition, the higher the dependence of the individual scores, the lower life satisfaction evaluation, as an inverse relationship. This is because the high scores in dependence scale prompt individual psychological maladjustment. It is performance in which has a strong dependence to others, often feel misunderstood by others, the mood is always unpleasant, and the body uncomfortable and so on. When individuals appeared bad adaptation to the environment, trust no one and always feeling troubles, this suggests us may be related to strong dissatisfaction of life. The result is consistent with the study of theoretical assumptions.

\section{Conclusions}

\subsection{Research Results}

High school students showed a moderate level of life satisfaction and in the performance of the differences between science and arts; the life satisfaction of science students was slightly higher than that of liberal arts students. Regression analysis showed that the degree of family care and psychological dependence can effectively predict life satisfaction. That is to say, family care positively predicted life satisfaction, psychological dependence on the role of negative prediction.

\subsection{Improvement Measures}

According to this study, it is concluded that the improvement of the individual's life satisfaction degree should be based on the improvement of the emotional care of the family members and the psychological dependence of the correction (Sun \& Yang, 2009). A person who is satisfied with the environment of his life, at the same time, has a good attitude to be possible to improve their inner happiness. Combined with the current high school students living and learning environment, we believe that in order to improve high school students life satisfaction in addition to optimizing the social environment, we also can do it from the following several aspects: 1) Schools can set up psychological activity course, to help students better self-awareness, self acceptance, correct the bad habits of self and in groups to find happiness, find their own advantages and strengths and avoid because of inferiority caused by bad mood; 2) in the school's study and life, we can promote the exchange of different members by using flexible classroom, as a way to encourage different gender and different family backgrounds students to publish more than their own opinions. With the way of learning, students can find their own shortcomings, and learn others' behavior as an example. Let the students in the exchange of the group gradually grow, to correct bad habits and attitudes; 3) as parents, they should be more communication with their children and listen to the views or ideas of children and the child's problems should have the patience to guide. Improving the life satisfaction of high school students will help them to better adapt to the society and can better play their own advantages; 4) parents whose children of different gender should with the same attitude to the education. For girls parents should cultivate the independent ability, for boys in cultivating independence at the same time parents should also be more care and greetings. If we do the above aspects, we can create a warm and harmonious family atmosphere and cultivate mental health and positive thinking of a new generation of young people and social harmony and stability development.

\subsection{Insufficient Research and Prospect}

There are many deficiencies in this research; for example the research sample is too small and the sample is not wide. It may be due to the limitation of the sample cause the results of deviation, so the research results can not be widely used. Previous studies on the relationship between life satisfaction and individual personality change in this area are not many. We can found in the theory of relatively narrow face. There are many factors that affect life satisfaction, and the factors of this study are not enough to summarize all of the factors, so the results of the study need to be further demonstrated. But the study results for us to improve the senior high school students' life satisfaction are a good reminder. It also tells us that family relationships influence the child's life with a 
large extent, that is to say family relationship is very important to the healthy growth of children. We hope that in the future time, there is more research theory support.

\section{References}

Chen, L. N., \& Zhang, J. X. (2005). College Students' General Life Satisfaction and Its Relationship with Self-Esteem. Chinese Journal of Mental Health, 18, 222-224.

Hai, H. (2007). The Care of the Family Obsessive-Compulsive Disorder. Journal of health abstract, 3, 13-13.

He, G. H., \& Huang, C. Y. (2009). College Students' Self-Acceptance and Family Care of Related Research. Chinese Journal of Health Psychology, 5, 582-584.

Li, L. J. (2013). College Students’ Cognitive Style, the Relationship between Parents Upbringing and Life Satisfaction Research. Master Thesis of Qinghai Normal University.

Liu, W. (2006). Senior High School Students' Life Satisfaction of the Urban and Rural Differences. Chinese Journal of Mental Health, 20, 647-649.

Ma, Y. (2009). Ezhou Youth Life Satisfaction and the Relationship between Psychological Sub-Health State. Huazhong University of Science and Technology.

Miao, Y. J. (2007). Happiness: Indicators and Measurement. Guangdong Academy of Social Sciences, 3, 63-68.

Peng, G. F. (2012). Parents Teaching Way and Children of the Dependency Relationship Analysis. Journal of Chongqing Electronic Engineering Vocational College, 21, 101-104.

Sun, L. R., \& Yang, Z. L. (2009). Bias of Society’s Personality Factor Research Review. Journal of Psychological Science, 32, 630-632.

Tian, L. L., \& Zheng, X. (2008). Senior High School Students’ Five Factors of Personality and the Relation of Multidimensional Life Satisfaction. Chinese Journal of Mental Health, 21, 165-168.

Wang, J. X., \& Wang, J. C. (2006). Senior High School Students' General Life Satisfaction and the Relationship between Family Factors Research. Journal of Psychology and Behavior Research, 3, 301-304.

Yao, B. X., Shi, S. Q., \& Fang, S. H. (2011). Life Satisfaction Research Status and Prospect. Journal of Academia, 8, 218-227.

Zhang, Z. J. (2005). Behavioral Medicine Inventory Handbook. China Medical Electronic Audio and Video Publishing House, 163-166.

Zhao, H. X., \& Xi, W. G. (2011). College Students' State of the Economy and Anxiety Influence on Life Satisfaction. Journal of Chifeng Institute: Natural Science Edition, 4, 225-226. 